33. Kruglyak, Yu. A. (2014). Lessons of ach. Physics in Higher Education, 20 (1), 39-43. nanoelectronics. 5. Phonon transport in «bottom - up» appro-

Рекомендовано до публікаиії д-р фіз.-мат. наук Глушков О.В. Дата надходження рукопису 14.01.2015

Кругляк Юрий Алексеевич, доктор химических наук, профессор, кафедра информационных технологий, Одесский государственный экологический университет, ул. Львовская, 15, г. Одесса, Украина, 65016

E-mail: quantumnet@yandex.ua

УДК 537.32

DOI: $10.15587 / 2313-8416.2015 .36443$

\title{
ГРАФЕН В ТРАНСПОРТНОЙ МОДЕЛИ ЛАНДАУЭРА-ДАТТЫ-ЛУНДСТРОМА
}

\author{
(C) Ю. А. Кругляк
}

Обсуждаются такие свойства графена как плотность электронных состояний и носителей тока, число мод и максимальная проводимость, рассеяние и подвижность в графене, циклотронная частота и эффективная масса, плотность фононных состояний, сравнительный вклад электронов и фононов в теплопроводность графена. В справочных целях дается сводка термоэлектрических коэффициентов для графена в баллистическом и диффузионном режимах проводимости со степенным законом рассеяния

Ключевые слова: нанофизика, наноэлектроника, графен, число мод, максимальная проводимость, эффективная масса, фононные состояния, теплопроводность, термоэлектрические коэффициенть

There are discussed the following properties of graphene such as the density of electronic states and current carriers, the number of modes and maximum conductivity, scattering and mobility in graphene, the cyclotron frequency and the effective mass, phonon density of states, the relative contribution of electrons and phonons in the thermal conductivity of graphene. For reference purposes a summary of thermoelectric coefficients for graphene in ballistic and diffusive conduction regimes with the power law of scattering is given

Keywords: nanophysics, nanoelectronics, graphene, mode numbers, maximum conductivity, effective mass, phonon states, thermal conductivity, thermoelectric coefficients

\section{1. Введение}

Модель электронного транспорта ЛандауэраДатты-Лундстрома (ЛДЛ) в режиме линейного отклика сводится к паре транспортных уравнений [1]

$$
\begin{aligned}
& J_{x}=\sigma \frac{d\left(E_{F} / q\right)}{d x}-S \sigma \frac{d T}{d x}\left[A / \mathrm{M}^{2}\right], \\
& J_{Q x}=\pi \sigma \frac{d\left(E_{F} / q\right)}{d x}-\kappa_{0} \frac{d T}{d x}\left[\mathrm{Bm} / \mathrm{M}^{2}\right.
\end{aligned}
$$

или в обращенной форме

$$
\begin{gathered}
\frac{d\left(E_{F} / q\right)}{d x}=\rho J_{x}+S \frac{d T}{d x}, \\
J_{Q x}=\pi J_{x}-\kappa \frac{d T}{d x}
\end{gathered}
$$

c транспортными коэффициентами для 2D проводников

$$
\begin{gathered}
\sigma=1 / \rho=\int \sigma^{\prime}(E) d E \\
\sigma^{\prime}(E)=\frac{2 q^{2}}{h} \frac{M(E)}{W} \lambda(E)\left(-\frac{\partial f_{0}}{\partial E}\right),
\end{gathered}
$$

$$
\begin{aligned}
S & =-\frac{k}{q} \frac{1}{\sigma} \int\left(\frac{E-E_{F}}{k T}\right) \sigma^{\prime}(E) d E=\frac{s_{T}}{\sigma}=\frac{\pi}{T}, \\
\kappa_{0} & =T\left(\frac{k}{q}\right)^{2} \int\left(\frac{E-E_{F}}{k T}\right)^{2} \sigma^{\prime}(E) d E=\kappa+\pi S \sigma .
\end{aligned}
$$

Уравнения (1)-(8) справедливы для диффузионных резисторов любой размерности и любого масштаба, любого типа проводимости, включая биполярную проводимость. Уравнение (6) выписано для 2D проводника; это видно по множителю $M(E) / W$, где $W$ - ширина 2D проводника. При рассмотрении 3D проводников этот множитель нужно заменить на $M(E) / A$, где $A-$ площадь поперечного сечения 3D проводника, а для 1D проводника заменить просто на число мод проводимости $M(E)$. При рассмотрении транспорта во всех режимах от диффузионного до баллистического среднюю длину свободного пробега $\lambda(E)$ нужно заменить на кажущуюся длину по уравнению $1 / \lambda^{a p p}=1 / \lambda+1 / L$, где $L \quad-$ длина проводника. Фактически в этих уравнениях нужно задаться только двумя параметрами, $M(E)$ и $\lambda(E)$. Число мод для проводников любой размерности с параболической дисперсией определяется форму- 
лами (72)-(74) в [2], но сами уравнения (1)-(8) справедливы для любой зонной структуры, нужно лишь корректно задать число мод проводимости. Зависимость $\lambda(E)$ нужно определить в соответствии с характером рассеяния в проводнике; часто используется степенной закон рассеяния [1].

В настоящем обзоре рассматривается применение транспортной модели ЛДЛ к графену.

\section{2. Обзор элементарной теории графена}

Пожалуй, сегодня нет другого объекта, который вызывал бы столь пристальное и широкое внимание физиков, химиков и технологов, как графен. Опубликованы подробные обзоры по физике графена [3-9]. В приближении эффективной массы поведение электрона в графене дается уравнением Дирака - Вейля для безмассовых нейтрино [10-12]. В такой экзотической электронной системе особый интерес вызывают ее транспортные свойства [13-17]. После демонстрации получения образцов графена микромеханическим методом расщепления графита [18] качество образцов графена оказалось настолько высоким, что удалось реализовать баллистический транспорт на этих образцах $[18,19]$ и наблюдать квантовый эффект Холла, что в свою очередь явилось фактическим подтверждением справедливости «нейтринной» трактовки электронных состояний графена $[20,21]$. Это обстоятельство послужило «спусковым крючком» взрывного интереса к всесторонным исследованиям графена вплоть до включения расчетов зонной структуры графена в университетские курсы, например, в Стенфордском университете [22] и в Университете Пердью [23-25].

Вначале мы дадим сжатое изложение простейшей $\pi$-модели зонной структуры графена в рамках теории сильной связи [26] и приведем необходимые сведения о его зонной структуре, что послужит нам основой для расчета транспортных и не только свойств графена в модели ЛДЛ. В Приложении дадим сводку всех термоэлектрических свойств графена как в баллистическом, так и в диффузионном режиме.

Кристаллическая решетка графена представляет собой совокупность двух взаимно-проникающих решеток Бравэ А и В с элементарной ячейкой в виде правильного ромба (рис. 1, 2). Период этих решеток равен $a=a_{0} \sqrt{3} \approx 2.46 A$, где длина связи СС $a_{0}=1.42 A$. Зона Бриллюэна представляет собой правильный шестиугольник со стороной $4 \pi / 3 a$ (Приложение I). Примитивные векторы трансляции (рис. 2) выбраны следующим образом: $\mathbf{a}=a(1,0)$ и $\mathbf{b}=a(-1 / 2, \sqrt{3} / 2)$.

Векторы, связывающие соседние атомы, равны $\boldsymbol{\tau}_{1}=a(0,1 / \sqrt{3}), \quad \boldsymbol{\tau}_{2}=a(-1 / 2,-1 / 2 \sqrt{3})$, $\boldsymbol{\tau}_{3}=a(1 / 2,-1 / 2 \sqrt{3})$. Вектора обратной решетки равны $\mathbf{a}^{*}=(2 \pi / a)(1,1 / \sqrt{3}) \quad$ и $\quad \mathbf{b}^{*}=(2 \pi / a)(0,2 / \sqrt{3})$. Первая зона Бриллюэна имеет две узловые точки К и $\mathrm{K}^{\prime}$. Соответствующие волновые векторы даются выражениями

$\mathbf{K}=(2 \pi / a)(-2 / 3,0) \quad$ и

$\mathbf{K}^{\prime}=(2 \pi / a)(2 / 3,0)$. Далее

$\exp \left(i \mathbf{K} \cdot \boldsymbol{\tau}_{1}\right)=\omega, \quad \exp \left(i \mathbf{K} \cdot \boldsymbol{\tau}_{2}\right)=\omega^{-1}, \quad \exp \left(i \mathbf{K} \cdot \boldsymbol{\tau}_{3}\right)=1$,

$\exp \left(i \mathbf{K}^{\prime} \cdot \boldsymbol{\tau}_{1}\right)=1, \quad \exp \left(i \mathbf{K}^{\prime} \cdot \boldsymbol{\tau}_{2}\right)=\omega^{-1}, \quad \exp \left(i \mathbf{K}^{\prime} \cdot \boldsymbol{\tau}_{3}\right)=\omega$,

где $\omega=\exp (2 \pi i / 3)$ и удовлетворяет условию $1+\omega+\omega^{-1}=0$.

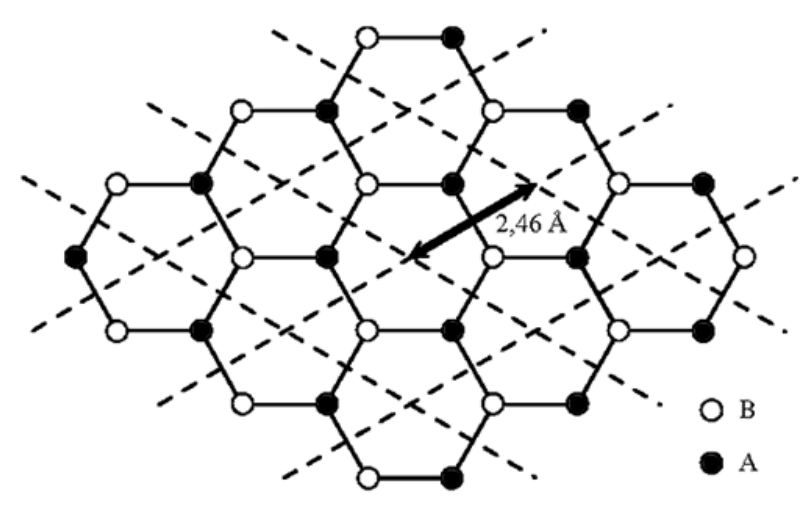

Рис. 1. Элементарная ячейка графена и вектор трансляции решеток Бравэ А и В

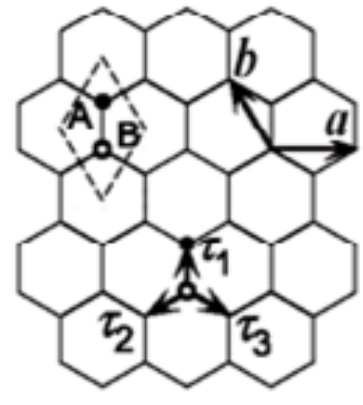

$a$

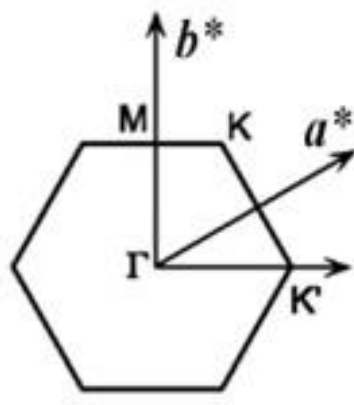

б
Рис. 2. Решетка графена ( $a$ ) и его первая зона Бриллюэна (б)

Пусть $\varphi(\mathbf{r})$ обозначает $2 p_{z}$-АО атома углерода C. Периодический потенциал решетки создается двумя смещенными друг относительно друга подрешетками А и В. Волновая функция электрона в таком потенциале дается линейной комбинацией двух блоховских волн, построенных на этих подрешетках

$\psi(\mathbf{r})=\sum_{\mathbf{R}_{A}} \psi_{A}\left(\mathbf{R}_{A}\right) \varphi\left(\mathbf{r}-\mathbf{R}_{A}\right)+\sum_{\mathbf{R}_{B}} \psi_{B}\left(\mathbf{R}_{B}\right) \varphi\left(\mathbf{r}-\mathbf{R}_{B}\right),(10)$

где амплитуды $\psi_{A}\left(\mathbf{R}_{A}\right)$ и $\psi_{\mathbf{B}}\left(\mathbf{R}_{B}\right)$ берутся в точках $\mathbf{R}_{A}=n_{a} \mathbf{a}+n_{b} \mathbf{b}+\boldsymbol{\tau}_{1} \quad$ и $\quad \mathbf{R}_{B}=n_{a} \mathbf{a}+n_{b} \mathbf{b}$ при целочисленных значениях $n_{a}$ и $n_{b}$. Пренебрежем интегралом перекрывания двух соседних АО $\left(S\left[2 p_{z}(A), 2 p_{z}(B)\right]=0\right) \quad$ и учтем резонансный интеграл только между соседними атомами 
$\left(\gamma_{0} \approx 3\right.$ э $\left.B\right)$, что позволит получить все решения в аналитическом виде. Тогда

$$
\begin{aligned}
& E \psi_{A}\left(\mathbf{R}_{A}\right)=-\gamma_{0} \sum_{l=1}^{3} \psi_{B}\left(\mathbf{R}_{A}-\boldsymbol{\tau}_{l}\right), \\
& E \psi_{B}\left(\mathbf{R}_{B}\right)=-\gamma_{0} \sum_{l=1}^{3} \psi_{A}\left(\mathbf{R}_{B}+\boldsymbol{\tau}_{l}\right),
\end{aligned}
$$

где за ноль отсчета энергии принята энергия $2 p_{z}$-АО атома С.

Положив $\quad \psi_{A}\left(\mathbf{R}_{A}\right)=f_{A}(\mathbf{k}) \exp \left(i \mathbf{k} \cdot \mathbf{R}_{A}\right) \quad$ и $\psi_{B}\left(\mathbf{R}_{B}\right)=f_{B}(\mathbf{k}) \exp \left(i \mathbf{k} \cdot \mathbf{R}_{B}\right)$, получим

$$
\begin{gathered}
\left(\begin{array}{cc}
0 & h_{A B}(\mathbf{k}) \\
h_{A B}(\mathbf{k}) * & 0
\end{array}\right)\left(\begin{array}{l}
f_{A}(\mathbf{k}) \\
f_{B}(\mathbf{k})
\end{array}\right)=E\left(\begin{array}{l}
f_{A}(\mathbf{k}) \\
f_{B}(\mathbf{k})
\end{array}\right), \\
h_{A B}(\mathbf{k})=-\gamma_{0} \sum_{l=1}^{3} \exp \left(-i \mathbf{k} \cdot \boldsymbol{\tau}_{l}\right) .
\end{gathered}
$$

Тогда для энергии зон имеем

$$
E_{ \pm}(\mathbf{k})= \pm \gamma_{0}\left|\sum_{l=1}^{3} \exp \left(-i \mathbf{k} \cdot \boldsymbol{\tau}_{l}\right)\right| .
$$

В соответствии с уравнением (10) имеем $E_{ \pm}(\mathbf{K})=E_{ \pm}\left(\mathbf{K}^{\prime}\right)=0$, т. е. в так называемых дираковских точках $K$ и $K^{\prime}$ отсутствует щель между валентной зоной $\pi$ и зоной проводимости $\pi^{*}$ (рис. 3 ).

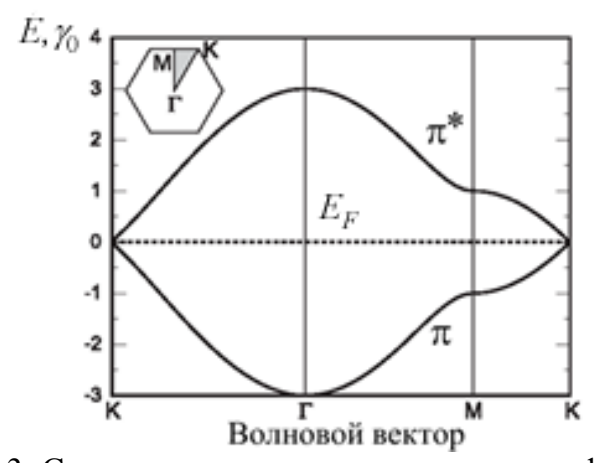

Рис. 3. Симметричная зонная структура графена в приближении Хюккеля:(S=0; $\left.\gamma_{0} \neq 0\right)$.

Посмотрим на поведение энергии в окрестности точки $K$. Перепишем $\mathbf{k}$ как $\mathbf{K}+\mathbf{k}$ и разложим энергию $E_{ \pm}(\mathbf{k})$ по степеням $|\mathbf{k}| a$. В наинизшем, первом порядке получим

$$
\begin{array}{r}
-\gamma_{0} \sum_{l=1}^{3} \exp \left[-i(\mathbf{K}+\mathbf{k}) \cdot \boldsymbol{\tau}_{l}\right]=-\omega^{-1} \gamma\left(k_{x}-i k_{y}\right) \\
\gamma=\frac{\sqrt{3}}{2} a \gamma_{0} .
\end{array}
$$

Поэтому когда мы переопределяем $f_{A}(\mathbf{K}+\mathbf{k})=\tilde{f}_{A}(\mathbf{k})$ и $f_{B}(\mathbf{K}+\mathbf{k})=-\omega \tilde{f}_{B}(\mathbf{k})$, имеем

$$
\gamma\left(\begin{array}{cc}
0 & k_{x}-i k_{y} \\
k_{x}+i k_{y} & 0
\end{array}\right)\left(\begin{array}{c}
\tilde{f}_{A}(\mathbf{k}) \\
\tilde{f}_{B}(\mathbf{k})
\end{array}\right)=E\left(\begin{array}{c}
\tilde{f}_{A}(\mathbf{k}) \\
\tilde{f}_{B}(\mathbf{k})
\end{array}\right)
$$

Используя матрицы Паули

$$
\sigma_{x}=\left(\begin{array}{ll}
0 & 1 \\
1 & 0
\end{array}\right), \quad \sigma_{y}=\left(\begin{array}{cc}
0 & -i \\
i & 0
\end{array}\right), \quad \sigma_{z}=\left(\begin{array}{cc}
1 & 0 \\
0 & -1
\end{array}\right),
$$

предыдущее уравнение (16) можно переписать в виде

$$
\gamma(\boldsymbol{\sigma} \cdot \mathbf{k}) \tilde{\mathbf{f}}(\mathbf{k})=E \tilde{\mathbf{f}}(\mathbf{k}), \quad \tilde{\mathbf{f}}(\mathbf{k})=\left(\begin{array}{c}
\tilde{f}_{A}(\mathbf{k}) \\
\tilde{f}_{B}(\mathbf{k})
\end{array}\right),
$$

где $\boldsymbol{\sigma}=\left(\sigma_{x}, \sigma_{y}\right)$. Аналогичным образом получаем уравнение для точки $K^{\prime}$. Фактически, достаточно лишь $\boldsymbol{\sigma}$ заменить на $\boldsymbol{\sigma} *$.
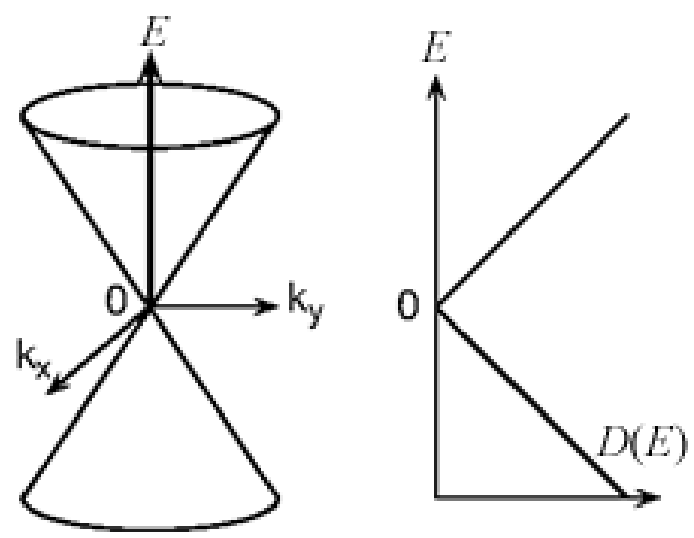

Рис. 4. Дисперсия энергии и плотность состояний $D(E)$ в окрестности точек К и $\mathrm{K}^{\prime}$

Таким образом, в окрестностях точек $K$ и $K^{\prime}$ дисперсия энергии дается простым выражением

$$
E_{s}(\mathbf{k})=s \gamma|\mathbf{k}|, \quad s= \pm 1,
$$

а плотность состояний равна

$$
D(E)=\frac{g_{v} g_{s}}{L^{2}} \sum_{s, k} \delta(E-s \gamma|\mathbf{k}|)=\frac{g_{v} g_{s}|E|}{2 \pi \gamma^{2}},
$$

где $L_{x} L_{y}-$ площадь графена, вырождение по спину $g_{s}=2$, а наличие двух долин $K$ и $K^{\prime}$ приводит к пространственному (долинному) вырождению $g_{v}=2$. Полученные результаты иллюстрируются рис. 4. Плотность состояний исчезает в точке $E=0$. Поэтому графен часто рассматривают как двумерный полупроводник с нулевой запрещенной зоной или же как полуметал.

В рассматриваемом приближении эффективной массы, иначе, схемы $\mathbf{k} \cdot \mathbf{p}$, движение электрона вблизи точек $K$ и $K^{\prime}$ описывается уравнением Шредингера, в котором вектор $\mathbf{k}$ заменяется оператором $\hat{\mathbf{k}}=-i \vec{\nabla}$. Имеем 


$$
\begin{aligned}
& \gamma(\boldsymbol{\sigma} \cdot \hat{\mathbf{k}}) \mathbf{F}^{K}(\mathbf{r})=E \mathbf{F}^{K}(\mathbf{r}), \\
& \gamma(\boldsymbol{\sigma} * \hat{\mathbf{k}}) \mathbf{F}^{K^{\prime}}(\mathbf{r})=E \mathbf{F}^{K^{\prime}}(\mathbf{r}),
\end{aligned}
$$

где $\mathbf{F}^{K}(\mathbf{r})$ и $\mathbf{F}^{K^{\prime}}(\mathbf{r})$ являются двухкомпонентной волновой функцией

$$
\mathbf{F}^{K}(\mathbf{r})=\left(\begin{array}{l}
F_{A}^{K}(\mathbf{r}) \\
F_{B}^{K}(\mathbf{r})
\end{array}\right), \quad \mathbf{F}^{K^{\prime}}(\mathbf{r})=\left(\begin{array}{l}
F_{A}^{K^{\prime}}(\mathbf{r}) \\
F_{B}^{K^{\prime}}(\mathbf{r})
\end{array}\right) .
$$

Уравнения (21) - это ничто иное как уравнения Дирака-Вейля для нейтрино, исключая разве что изотропную скорость

$$
v_{F}=\frac{1}{\hbar} \frac{\partial E}{\partial k}=\frac{\gamma}{\hbar},
$$

меньшую скорости света всего приблизительно в 300 раз. Столь высокая скорость электронов в графене привлекла внимание специалистов по наноэлектронике.

Итак, вблизи точки Дирака

$$
E(k)= \pm \hbar v_{F} k= \pm \hbar v_{F} \sqrt{k_{x}^{2}+k_{y}^{2}} .
$$

Графен с $E_{F}>0$ назовем $n$-проводником, а с $E_{F}<0-p$-проводником. Щели между зоной проводимости и валентной зоной нет. Обе зоны всегда спарены (рис. 5).

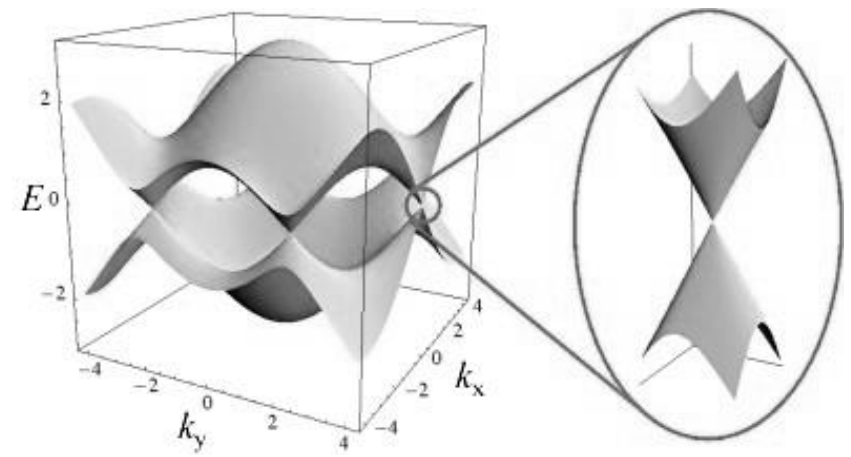

Рис. 5. Зонная структура графена. Увеличенным масштабом показан линейный спектр носителей тока в точке Дирака

Электроны в графене описываются двухкомпонентной волновой функцией

$$
\left(\begin{array}{l}
\psi_{A} \\
\psi_{B}
\end{array}\right)=\frac{1}{\sqrt{2}} \frac{1}{\sqrt{L_{x} L_{y}}}\left(\begin{array}{c}
1 \\
s e^{i \theta}
\end{array}\right) e^{i\left(k_{x} x+k_{y} y\right)},
$$

где $\theta=\operatorname{arctg}\left(k_{y} / k_{x}\right)$, что влечет за собой важные следствия; например, поскольку электроны вдоль $+k_{x}$ и $-k_{x}$ описываются ортогональными волновыми функциями, то вероятность обратного рассеяния на $180^{\circ}$ в графене нулевая.

\section{3. Плотность состояний и плотность носителей тока \\ В конечном итоге нужно вычислить проводимость графена. Проводимость зависит от}

$E_{F}$, а положение уровня Ферми зависит от плотности носителей тока, определяемой плотностью состояний $D(E)$.

Опуская подробности вычисления плотности состояний $[27,28]$, для 2D проводника число состояний между $k$ и $k+d k$

$$
N(k) d k=g_{s} g_{v} \frac{2 \pi k d k}{\left(2 \pi / L_{x}\right)\left(2 \pi / L_{y}\right)},
$$

где $L_{x} L_{y}=A$ есть площадь графена, для которой вычисляется число состояний. В знаменателе стоит площадь $k$-пространства, занятая $k$-состояниями. Для дисперсии (24)

$$
N(k) d k=g_{v} A \frac{E d E}{\pi\left(\hbar v_{F}\right)^{2}} .
$$

Определяя $D(E) d E$ как плотность состояний на единицу поверхности графена в интервале энергий между $E$ и $E+d E$ и учитывая, что энергия может быть как положительной, так и отрицательной, для плотности состояний графена окончательно получаем:

$$
D(E)=\frac{2|E|}{\pi \hbar^{2} v_{F}^{2}},
$$

а для образца графена с площадью поверхности $W \cdot L$ это удельное выражение нужно умножить на $W \cdot L$.

Число электронов в зоне проводимости

$$
n_{S}\left(E_{F}\right)=\int_{0}^{\infty} D(E) f_{0}(E) d E,
$$

где индексом $S$ пометим свойство, отнесенное к поверхности. Поскольку графен вырожден, то $T=0^{\circ} \mathrm{K}$ будет неплохим приближением даже при комнатной температуре. Уравнение (29) становится следующим

$$
n_{S}\left(E_{F}\right)=\int_{0}^{E_{F}} D(E) d E=\frac{2}{\pi \hbar^{2} v_{F}^{2}} \int_{0}^{E_{F}} d E,
$$

так что окончательно для плотности носителей тока имеем:

$$
n_{S}\left(E_{F}\right)=\frac{E_{F}^{2}}{\pi \hbar^{2} v_{F}^{2}} .
$$

\section{4. Число мод и проводимость}

Для 2D проводника число мод (ф-ла (63) в [2])

$$
M(E)=W M_{2 D}(E)=W \frac{h}{4}\left\langle v_{x}^{+}(E)\right\rangle D_{2 D}(E),
$$

где $W$ - ширина проводника. Для графена

$$
\left\langle v_{x}^{+}(E)\right\rangle=\frac{2}{\pi} v_{F},
$$

плотность состояний дается формулой (28), так что для числа мод окончательно имеем:

$$
M(E)=W \frac{2|E|}{\pi \hbar v_{F}} .
$$


В отличии от параболической дисперсии, для которой поведение числа мод и плотности состояний с энергией различное [2], в графене оба эти свойства пропорциональны энергии:

$$
D(E), M(E) \propto E .
$$

Теперь можно сразу вычислить проводимость. Согласно (6) с учетом вырожденности $\left(T=0^{\circ} K\right)$,

$$
\begin{aligned}
& \sigma^{\prime}(E)=\frac{2 q^{2}}{h} \frac{M(E)}{W} \lambda(E)\left(-\frac{\partial f_{0}}{\partial E}\right)= \\
& =\frac{2 q^{2}}{h} \frac{M(E)}{W} \lambda(E) \delta\left(E-E_{F}\right),
\end{aligned}
$$

так что окончательно поверхностная проводимость (5)

$$
\sigma_{S}=\int \sigma^{\prime}(E) d E=\frac{2 q^{2}}{h}\left(\frac{2 E_{F}}{\pi \hbar v_{F}}\right) \lambda\left(E_{F}\right) .
$$

Для не зависящей от энергии средней длины свободного пробега проводимость графена пропорциональна энергии Ферми, а по (31) стало быть линейно зависит от $\sqrt{n_{S}}$.

\section{5. Рассеяние в графене}

Характер зависимости проводимости от энергии Ферми или от поверхностной плотности носителей тока диктуется зависимостью среднего пути свободного пробега от энергии $\lambda(E)$, который в свою очередь определяется временем релаксации импульса согласно [29]

$$
\lambda(E)=\frac{\pi}{2} v_{F} \tau_{p} .
$$

Там же показано, что для короткодействующих потенциалов рассеяния и рассеяния на акустических фононах скорость рассеяния пропорциональна плотности состояний:

$$
\frac{1}{\tau_{p}} \propto D(E) \propto E
$$

так что в итоге

$$
\tau_{p} \propto \lambda(E) \propto E^{-1}
$$

и не зависит от температуры. Согласно же (37), теперь нужно признать, что для короткодействующих потенциалов рассеяния и рассеяния на акустических фононах поверхностная проводимость $\sigma_{S}$ должна быть константой, не зависящей от $E_{F}$ или $n_{S}$. Поскольку

$$
\sigma_{S}=q n_{S} \mu,
$$

то подвижность $\mu$ должна быть обратно пропорциональна $n_{s}$. Это необычная ситуация. Ведь обычно чем больше плотность носителей тока, тем больше проводимость. Можно заключить, что если доминируют короткодействующие потенциалы рассеяния или рассеяние на акустических фононах, то можно ожидать независимости проводимости от $n_{S}[13]$ (рис. 6).

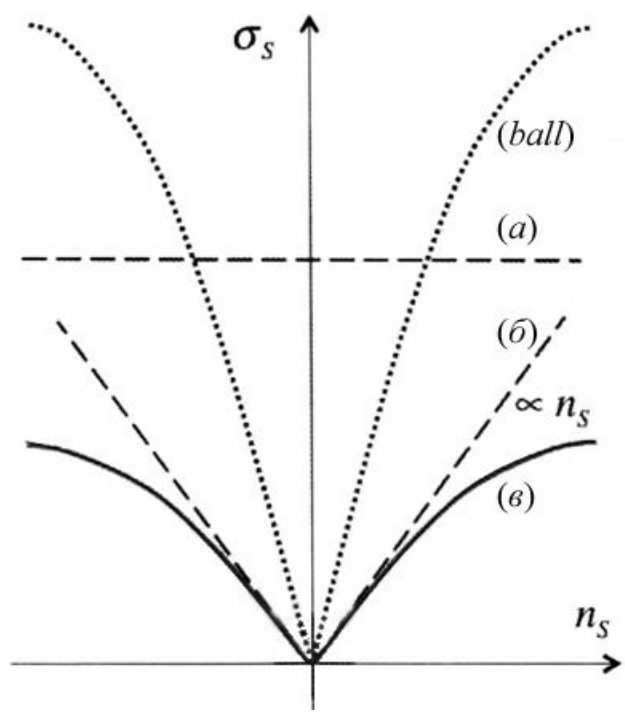

Рис. 6. Качественная картина зависимости проводимости от плотности носителей тока для графена: $a$ - для короткодействующих потенциалов рассеяния и рассеяния на акустических фононах

зависимость отсутствует; $\sigma$ - рассеяние на заряженных примесях дает линейную зависимость; в - суммарная картина; показан также баллистический режим

В случае рассеяния на экранированных и неэкранированных ионизированных примесях для $\lambda(E)$ можно ожидать линейную зависимость от энергии [15-17]

$$
\lambda(E)=\lambda_{i i} E,
$$

что после подстановки в (37) дает квадратичную зависимость от $E_{F}$. Плотность носителей тока (31) также квадратична по $E_{F}$. Таким образом, рассеяние на заряженных примесях ведет к проводимости, линейно зависящей от $n_{s}$ и, согласно (41), к постоянному значению подвижности [14].

На рис. 6 качественно иллюстрируются два рассмотренных выше механизма рассеяния: случай короткодействующих потенциалов рассеяния (sr/short range) и рассеяние на ионизированных примесях (ii/ionized impurities). Скорости рассеяния складываются, так что суммарное значение средней длины свободного пробега

$$
\frac{1}{\lambda_{t o t}}=\frac{1}{\lambda_{i i}}+\frac{1}{\lambda_{s r}}
$$

и

$$
\frac{1}{\sigma_{t o t}}=\frac{1}{\sigma_{i i}}+\frac{1}{\sigma_{s r}} .
$$

Для выбранного значения $n_{S}$ наименьший из двух вкладов лимитирует суммарное значение $\sigma_{S}$, 
из чего следует нелинейная суммарная зависимость $\sigma_{S}\left(n_{S}\right)$.

Еще несколько механизмов рассеяния могут оказаться важными в графене. Это, например, короткодействующее рассеяние на дефектах сотовой структуры графена и рассеяние на полярных оптических фононах в $\mathrm{SiO}_{2}$, часто служащем подложкой для графена [15-17].

\section{6. Подвижность и формула Друде}

Подвижность в графене находим уравниванием выражений (37) и (41):

$$
\mu=\frac{2 q}{h} \frac{1}{n_{S}}\left(\frac{2 E_{F}}{\pi \hbar v_{F}}\right) \lambda\left(E_{F}\right) .
$$

После подстановки выражения для $n_{S}$ получаем

$$
\mu=\frac{2 q v_{F}}{\pi E_{F}} \lambda\left(E_{F}\right) .
$$

Часто пишут, что электроны в графене «безмассовые». Это утверждение не лишено смысла, поскольку линейная дисперсия в графене (24) похожа на частотную дисперсию для фотонов $\omega(k)$. Соответственно этому для нахождения подвижности не стоит пользоваться формулой Друде с некой эффективной массой

$$
\mu=\frac{q \tau_{p}}{m^{*}},
$$

а пользоваться напрямую формулой (46). Вместе с тем, связь между двумя подходами можно установить. Для этого выразим длину свободного пробега в (46) через время релаксации по (38). Тогда

$$
\mu=\frac{q}{E_{F} / v_{F}^{2}} \tau_{p}\left(E_{F}\right),
$$

что напоминает формулу Друде для подвижности (47), если под так называемой эффективной массой в графене подразумевать

$$
m^{*}=\frac{E_{F}}{v_{F}^{2}},
$$

что напоминает релятивистское выражение для энергии $E=m c^{2}$.

\section{7. Циклотронная частота в графене}

При помещении графена в магнитное поле, направленное перпендикулярно его поверхности, циклотронная частота

$$
\omega_{c}=2 \pi / T,
$$

где $T$ - период обращения электрона по круговой орбите вокруг вектора магнитного поля (рис. 7)

$$
\vec{B}=B_{z} \hat{z}
$$

для слабых полей связана со средним временем свободного пробега $\tau_{p}$ соотношением

$$
\omega_{c} \tau_{p} \ll 1
$$

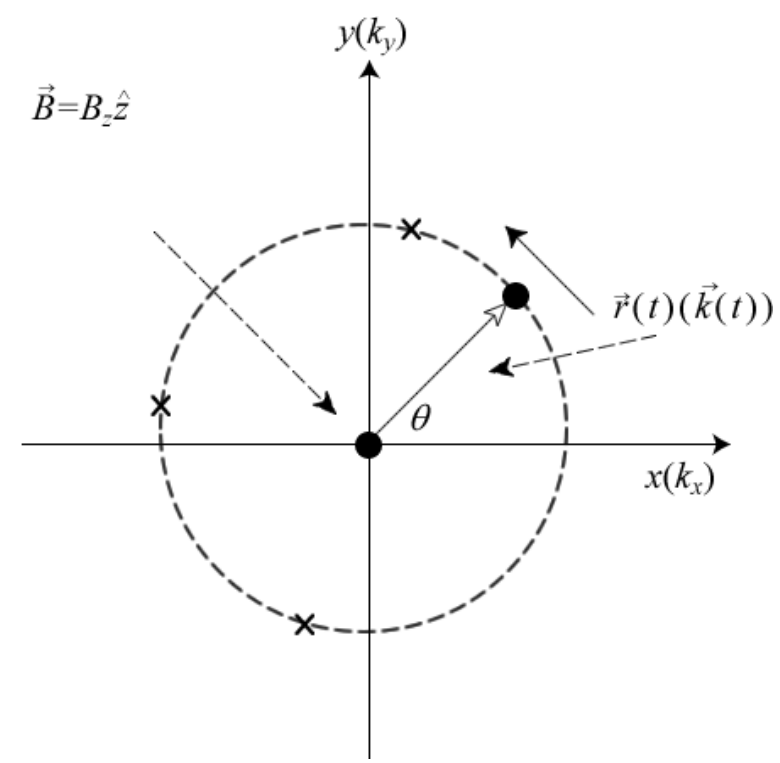

Рис. 7. К вычислению циклотронной частоты. Крестиком на траектории отмечены акты рассеяния

Для параболической дисперсии, как известно [30]

$$
\omega_{c}=\frac{q B_{z}}{m^{*}} .
$$

Тогда условие для слабых полей (52) с учетом (47) можно переписать еще так:

$$
\mu B_{z} \ll 1 .
$$

Из (50) и (52) следует, что для слабых полей

$$
T \gg \tau_{p},
$$

другими словами, период обращения электрона намного превышает время между столкновениями, в результате чего лишь редкие орбиты оказываются замкнутыми, что и приводит к наблюдению классического эффекта Холла. В противном случае, когда

$$
\omega_{c} \tau_{p} \gg 1
$$

что соответствует сильным магнитным полям, наблюдается квантовый эффект Холла.

Получим общее выражение для циклотронной частоты, пригодное для произвольной дисперсии. Для силы Лоренца, действующей на электрон в магнитном поле, имеем

$$
\frac{d \vec{p}}{d t}=-q \vec{v} \times \vec{B}
$$

Учтем, что импульс

$$
\vec{p}=\hbar \vec{k},
$$

и распишем (57) покомпонентно: 


$$
\begin{aligned}
& \hbar \frac{d k_{x}}{d t}=-q v_{y} B_{z} \\
& \hbar \frac{d k_{y}}{d t}=+q v_{x} B_{z},
\end{aligned}
$$

что дает (рис. 7):

$$
\begin{aligned}
& \hbar k \frac{d(\cos \theta)}{d t}=-q(v \sin \theta) B_{z}, \\
& \hbar k \frac{d(\sin \theta)}{d t}=+q(v \cos \theta) B_{z} .
\end{aligned}
$$

Дифференцируя (59) по времени и используя (60), получаем

$$
\frac{d^{2}(\cos \theta)}{d t^{2}}=-\omega_{c}^{2} \cos \theta,
$$

где искомая циклотронная частота для изотропной зонной структуры

$$
\omega_{c}=\frac{q v}{\hbar k} B_{z} .
$$

Для параболической дисперсии

$$
v=\frac{\hbar k}{m^{*}},
$$

что и дает для этой дисперсии циклотронную частоту (53).

Для интересующего нас случая графена $v=v_{F}$ и $E=\hbar v_{F} k$ для циклотронной частоты в графене из (61) получаем

$$
\omega_{c}=\frac{q B_{z}}{E_{F} / v_{F}^{2}} .
$$

Таким образом, и здесь справедливо полученное ранее выражение (49) для эффективной массы.

\section{8. Эффективная масса в графене}

Выражение для эффективной массы в графене из подвижности (49) и циклотронной частоты (63) указывает на то, что должен быть более общий подход к понятию эффективной массы, чем традиционное определение ее через кривизну дисперсионного соотношения

$$
m^{*}=\hbar^{2} /\left(\frac{d^{2} E}{d k^{2}}\right)_{k=0} .
$$

Разные способы записи проводимости для 2D проводников приведены в [31]. В общем случае имеем два эквивалентных выражения для проводимости [32], одно из которых выражает проводимость через произведение плотности состояний $D(E)$ и коэффициента диффузии $\bar{D}$

$$
\sigma(E)=q^{2} \bar{D} \frac{D(E)}{L}\left\{1, \frac{1}{W}, \frac{1}{A}\right\},
$$

a другое - через произведение числа мод $M(E)$ в канале проводимости и средней длины пробега $\lambda(E)$

$$
\sigma(E)=\frac{q^{2}}{h} M(E) \lambda(E)\left\{1, \frac{1}{W}, \frac{1}{A}\right\},
$$

где выражение в фигурных скобках показывает множители для проводников разной размерности.

Применимость модели Друде ограничена, в то время как уравнения для проводимости (65) и (66) имеют самое общее значение. Фундаментальное различие между этими уравнениями и теорией Друде состоит в том, что усреднение (5), (6)

$$
\sigma=\int_{-\infty}^{+\infty} \sigma(E)\left(-\frac{\partial f_{0}}{\partial E}\right) d E
$$

делает проводимость свойством поверхности Ферми: проводимость определяется уровнями энергии, близкими к $E=E_{F 0}$. А согласно теории Друде проводимость зависит от общей электронной плотности, суммированной по всему спектру энергий, что и приводит к ограниченной применимости модели Друде. Проводимость веществ меняется в очень широких пределах несмотря на то, что число электронов в разных веществах приблизительно одинаково. Низкая проводимость стекла объясняется не тем, что в стекле мало так называемых «свободных» электронов, а потому, что для стекла характерна очень низкая плотность состояний и числа мод вблизи $E=E_{F 0}$. Понятие же «свободных» электронов относится к интуитивным понятиям.

Для произвольных проводников, как с кристаллической структурой, так и аморфных, так и для молекулярных проводников показано [32], что независимо от функциональной зависимости дисперсионного соотношения $E(p)$ плотность состояний $D(E)$, скорость $v(E)$ и импульс $p(E)$ связаны с числом электронных состояний $N(E)$ с энергией, меньшей значения $E$, фундаментальным соотношением

$$
D(E) v(E) p(E)=N(E) \cdot d,
$$

где $d$ - размерность проводника. Используя (68) для вычисления проводимости (65) с учетом коэффициента диффузии [33]

$$
\bar{D}=\left\langle v_{x}^{2} \tau_{p}\right\rangle
$$

для $3 \mathrm{~d}$-проводника получим

$$
\sigma(E)=q^{2} \frac{N(E)}{A \cdot L} \frac{\tau_{p}(E)}{m(E)} .
$$

где масса определена как

$$
m(E)=\frac{p(E)}{v(E)} .
$$

Легко убедиться, что фундаментальное соотношение (68) справедливо и для параболической дисперсии и для линейной. Для параболической зависимости масса носителей тока от энергии не зависит, что в общем случае не так.

Формула (70) выглядит как в теории Друде, если $N / A \cdot L$ считать электронной плотностью $n$. При 
низких температурах это действительно так, поскольку усреднение (67) при $E=E_{F 0}$ дает

$$
\sigma=\left(q^{2} \frac{N(E)}{A \cdot L} \frac{\tau_{p}}{m}\right)_{E=E_{F 0}}=q^{2} n \tau_{p} / m,
$$

так как $N(E)$ при $E=E_{F 0}$ есть полное число электронов. При ненулевой температуре ситуация тем более сложнее, поскольку плотность состояний непараболическая. Отметим, что ключевым моментом в сведении общего выражения для проводимости (65) к выражению (70), похожему на формулу Друде, есть фундаментальное соотношение (68), связывающее плотность состояний $D(E)$, скорость $v(E)$ и импульс $p(E)$ для данного значения энергии с полным числом состояний $N(E)$, полученных интегрирование плотности состояний

$$
N(E)=\int_{-\infty}^{E} D(E) d E .
$$

\section{9. Плотность фононных состояний} графене

Плотность фононных состояний и фононные моды для проводников разной размерности подробно рассмотрены в [34]. В качестве примера обратимся к вычислению плотности состояний для фононных акустических мод в графене, ход которых качественно показан на рис. 8 .

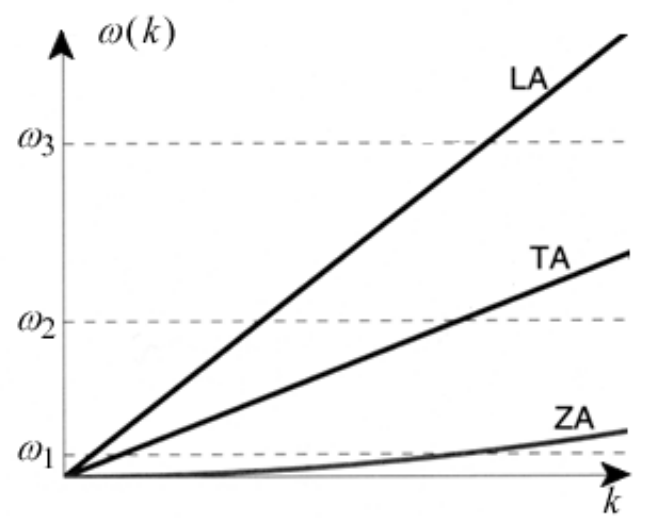

Рис. 8. К вычислению плотности фононных состояний на разных частотах

Вычислим плотность фононных состояний для конкретных частот $\omega_{1}, \omega_{2}$ и $\omega_{3}$. Если продольные LA и поперечные ТА акустические моды можно аппроксимировать линейной зависимостью $\omega(k)$, а именно:

$$
\begin{aligned}
& \omega=v_{g}^{\mathrm{LA}} k, \\
& \omega=v_{g}^{\mathrm{TA}} k,
\end{aligned}
$$

то изгибные моды (flexural/ZA) ближе всего аппроксимируются квадратичной зависимостью частоты

$$
\omega \propto \text { Const }^{Z A} \cdot k^{2},
$$

где для графена $[35,36]$

$$
\begin{gathered}
v_{g}^{\mathrm{LA}} \approx 2 \times 10^{4}, v_{g}^{\mathrm{TA}} \approx 1.5 \times 10^{4} \mathrm{M} / \mathrm{c}, \\
\text { Const }^{\mathrm{ZA}} \approx 5 \times 10^{-7} \mathrm{M}^{2} / \mathrm{c} .
\end{gathered}
$$

Подстановка (74) в (62) работы [34] окончательно для плотности фононных LA мод дает

$$
D_{2 D}^{L A}(\omega)=\frac{\omega}{2 \pi\left(v_{g}^{\mathrm{LA}}\right)^{2}}
$$

и аналогично для ТА мод

$$
D_{2 D}^{T A}(\omega)=\frac{\omega}{2 \pi\left(v_{g}^{\mathrm{TA}}\right)^{2}} .
$$

Для ZА мод с учетом (59) работы [34] и (74) окончательно имеем

$$
D_{2 D}^{Z A}(\omega)=\frac{1}{L^{2}} \frac{d N_{2 D}(k)}{d k} \frac{d k}{d \omega}=\frac{1}{4 \pi \cdot \text { Const }^{Z A}} .
$$

На частоте $\omega_{1}$ вклад в плотность фононных состояний дают все три моды (рис. 8):

$$
\begin{aligned}
& D_{2 D}^{\text {tot }}\left(\omega_{1}\right)=D_{2 D}^{L A}\left(\omega_{1}\right)+D_{2 D}^{T A}\left(\omega_{1}\right)+D_{2 D}^{Z A}\left(\omega_{1}\right)= \\
& =\frac{\omega_{1}}{2 \pi\left(v_{g}^{\mathrm{LA}}\right)^{2}}+\frac{\omega_{1}}{2 \pi\left(v_{g}^{\mathrm{TA}}\right)^{2}}+\frac{1}{4 \pi \cdot \text { Const }^{\mathrm{ZA}}},
\end{aligned}
$$

на частоте $\omega_{2}$ - только моды LA и TA:

$$
D_{2 D}^{t o t}\left(\omega_{2}\right)=D_{2 D}^{L A}\left(\omega_{2}\right)+D_{2 D}^{T A}\left(\omega_{2}\right)=\frac{\omega_{2}}{2 \pi\left(v_{g}^{\mathrm{LA}}\right)^{2}}+\frac{\omega_{2}}{2 \pi\left(v_{g}^{\mathrm{TA}}\right)^{2}},
$$

а на частоте $\omega_{3}$ - только мода LA:

$$
D_{2 D}^{t o t}\left(\omega_{3}\right)=D_{2 D}^{L A}\left(\omega_{3}\right)=\frac{\omega_{3}}{2 \pi\left(v_{g}^{\mathrm{LA}}\right)^{2}}
$$

10. Вклад электронов и фононов в теплопроводность графена

Уникальная зонная структура графена в точках Дирака порождает исключительно высокую электронную проводимость графена, что в свою очередь привело к множеству разнообразных предложений и идей по применению графена в различных и порой необычных электронных устройствах [37]. В отличие от большинства известных материалов линейность зонной структуры графена напоминает поведение длинноволновых акустических фононов. В отличие от исключительного внимания к электрическим свойствам графена его термические свойства менее изучены. Вместе с тем именно фононы ответственны за его теплофизические свойства. Приведем качественное объяснение причин такой ситуации. Для этого нужно обратиться к фононным модам.

Согласно (34), в случае электронов для плотности числа мод имеем

$$
M_{2 D}(E)=\frac{2|E|}{\pi \hbar v_{F}} .
$$


Для плотности числа фононов, согласно (67б) работы [34], имеем

$$
M_{2 D}(\omega)=2 v_{g}(\omega) D_{2 D}(\omega)=\frac{k(\omega)}{\pi} .
$$

Для LA и TA акустических фононов воспользуемся, согласно (74 a, б), линейным дебаевским приближением, а для ZА фононов квадратичной аппроксимацией (75). Тогда для плотности фононных состояний имеем:

$$
\begin{gathered}
M_{2 D}^{L A}(\omega)=\frac{\omega}{\pi v_{g}^{L A}}, \\
M_{2 D}^{T A}(\omega)=\frac{\omega}{\pi v_{g}^{T A}}, \\
M_{2 D}^{Z A}(\omega)=\frac{1}{\pi} \sqrt{\frac{\omega}{\text { Const }^{Z A}}} \\
\text { с параметрами } v_{g}^{L A}, v_{g}^{T A} \text { и } \text { Const }^{Z A} \text { по (75). }
\end{gathered}
$$

Сравнивая плотность числа электронных мод (78) с плотностью LA и TA фононных состояний (80), мы фактически имеем одно и то же выражение $(E=\hbar \omega)$ за исключением двойки, учитывающей вырождение по спину. Отличаются эти выражения лишь скоростью: скорость электронов $v_{F}$ на два порядка больше групповой скорости фононов $v_{g}$. В результате плотность электронных мод намного меньше плотности фононных мод. Это наглядно видно из рис. 9, где зависимость плотности всех акустических фононных мод отложена вместе с плотностью электронных мод в интервале $0<E \leq 50$ мэВ с максимальным значением, соответствующим $\approx 2 k T$ при комнатной температуре.

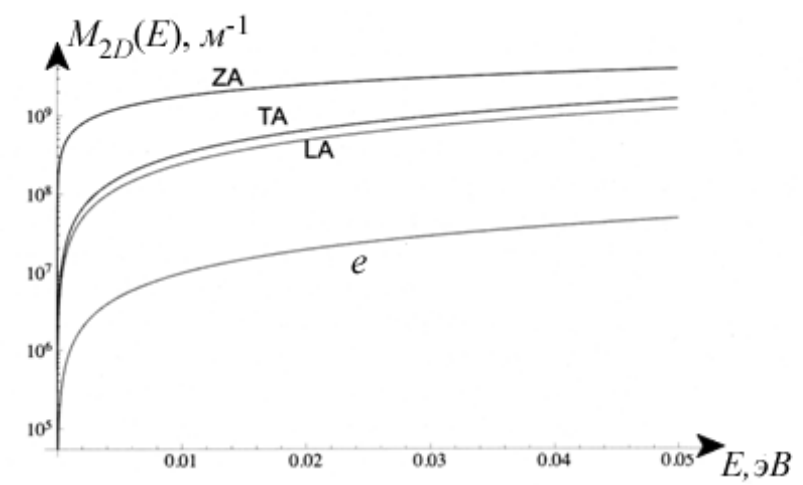

Рис. 9. Плотность электронных и фононных состояний в графене вплоть до 50 мэ $B$ [36]

Как следствие, нужно ожидать намного более существенный вклад фононов в передачу тепла по сравнению с электронами. А среди фононных акустических мод наиболее важную роль в передаче тепла играют флексюральные моды ZA [34] по крайней мере вплоть до комнатных температур.

\section{1. Максимальная проводимость графена}

Рассмотрим идеально чистый графен без дефектов и без заряженных примесей, рассеивающих электроны. Какую проводимость можно ожидать? При высоких температурах имеет место рассеяние на фононах, вблизи же комнатной температуры доминирует рассеяние на акустических фононах. Скорость рассеяния пропорциональна плотности состояний, а для времени рассеяния получено следующее выражение [28]:

$$
\tau_{p}(E)=\frac{4 \hbar^{3} \rho_{m} v_{F}^{2} v_{S}^{2}}{D_{A}^{2} k T}\left(\frac{1}{E}\right),
$$

где $\rho_{m}$ - массовая плотность; $v_{S}$ - скорость звука; $D_{A}$ - акустический деформационный потенциал, служащий мерой электрон-фононной связи. Тогда для среднего пути свободного пробега, согласно (38), получаем:

$$
\lambda(E)=\frac{2 \pi \hbar^{3} \rho_{m} v_{F}^{3} v_{S}^{2}}{D_{A}^{2} k T}\left(\frac{1}{E}\right)
$$

и далее из уравнения (37) для поверхностной проводимости имеем:

$$
\sigma_{S}=\frac{4 q^{2} \hbar \rho_{m} v_{F}^{2} v_{S}^{2}}{\pi D_{A}^{2} k T}\left(\frac{1}{E}\right) .
$$

Подставляя следующие значения свойств для графена [29]

$$
v_{S} \approx 2.1 \times 10^{4} \mathrm{M} / \mathrm{c}, \quad \rho_{m} \approx 7.6 \times 10^{-7} \mathrm{~K} / \mathrm{M}^{2}, \quad D_{A} \approx 18 \ni B,
$$
получаем

$$
\rho_{S}=\frac{1}{\sigma_{S}} \approx 30 O M / \square .
$$

Оценка (84) дает ожидаемый нижний предел для удельного поверхностного сопротивления (верхний предел для удельной поверхностной проводимости). В реальности другие механизмы рассеяния увеличат поверхностное сопротивление. Как соотносится полученная оценка с поверхностным сопротивлением для других материалов? Рассмотрим, например, Si MOSFET с $n_{S} \approx 10^{13} \mathrm{~cm}^{-3}$ и подвижностью в инверсионном слое $\mu_{\text {eff }} \approx 250 \mathrm{~cm}^{2} / B \cdot c$. Для такого 2D проводника $\rho_{S} \approx 2500 O M / \square$. Для высокопроводящего InGaAs c $n_{S} \approx 2 \times 10^{12} \mathrm{~cm}^{-3} \quad$ и подвижностью $\mu_{\text {eff }} \approx 1 \times 10^{5} \mathrm{~cm}^{2} / B \cdot c$ находим, что $\rho_{S} \approx 300 O M / \square$. Действительно, для графена можно ожидать исключительно высокую проводимость.

В заключение в справочных целях приводим в Приложении II термоэлектрические коэффициенты для графена в баллистическом и диффузионном режимах $[29,38]$.

\section{Приложение I. Обратная решетка и зона Бриллюэна графена}

Построим обратную решетку графена и его зону Бриллюэна. Примитивные векторы трансляции можно выбрать любым приемлемым способом. В отличие от выбора на рис. 2, пусть сейчас векторы трансляции выберем иначе (рис. 10). 


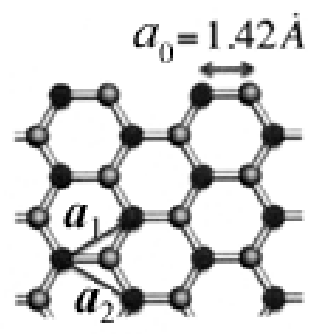

Рис. 10. Выбор примитивных векторов трансляции в решетке графена

Эти базисные векторы, очевидно, таковы:

$$
\begin{aligned}
& \mathbf{a}_{1}=\frac{3}{2} a_{0} \hat{x}+\frac{\sqrt{3}}{2} a_{0} \hat{y}, \\
& \mathbf{a}_{2}=\frac{3}{2} a_{0} \hat{x}-\frac{\sqrt{3}}{2} a_{0} \hat{y}, \\
& \mathbf{a}_{3}=c \hat{z},
\end{aligned}
$$

где вектор $\boldsymbol{a}_{3}$ играет вспомогательную роль: периодичность перпендикулярно плоскости графена отсутствует, величина этого вектора, как будет видно ниже, дается большим числом.

Произвольные векторы прямой

$$
\mathbf{R}=n_{1} \mathbf{a}_{1}+n_{2} \mathbf{a}_{2}+n_{3} \mathbf{a}_{3}
$$

и обратной

$$
\mathbf{G}=k_{1} \mathbf{b}_{1}+k_{2} \mathbf{b}_{2}+k_{3} \mathbf{b}_{3}
$$

решеток связаны соотношением:

$$
\mathbf{R} \cdot \mathbf{G}=2 \pi \times \operatorname{Int},
$$

где Int есть произвольное целое число, а трансляционные векторы обратной решетки

$$
\mathbf{b}_{i}=2 \pi \frac{\mathbf{a}_{j} \times \mathbf{a}_{k}}{\mathbf{a}_{1} \cdot\left(\mathbf{a}_{2} \times \mathbf{a}_{3}\right)},
$$

где знаменатель есть объем элементарной ячейки. Вычисляем векторы обратной решетки:

$$
\begin{aligned}
& \mathbf{b}_{1}=2 \pi \frac{\mathbf{a}_{2} \times \mathbf{a}_{3}}{\mathbf{a}_{1} \cdot\left(\mathbf{a}_{2} \times \mathbf{a}_{3}\right)}=\frac{2 \pi}{a_{0}}\left(\frac{1}{3} \hat{x}+\frac{1}{\sqrt{3}} \hat{y}\right) \\
& \mathbf{b}_{2}=2 \pi \frac{\mathbf{a}_{3} \times \mathbf{a}_{1}}{\mathbf{a}_{1} \cdot\left(\mathbf{a}_{2} \times \mathbf{a}_{3}\right)}=\frac{2 \pi}{a_{0}}\left(\frac{1}{3} \hat{x}-\frac{1}{\sqrt{3}} \hat{y}\right), \\
& \mathbf{b}_{3}=2 \pi \frac{\mathbf{a}_{1} \times \mathbf{a}_{2}}{\mathbf{a}_{1} \cdot\left(\mathbf{a}_{2} \times \mathbf{a}_{3}\right)}=\frac{2 \pi}{c} \hat{z}
\end{aligned}
$$

где $\left.\mathbf{b}_{3}\right|_{c \rightarrow \infty} \rightarrow 0$, что соответствует двумерности обратной решетки.

Относительно центра $Г$ первой зоны Бриллюэна строим векторы $\mathbf{b}_{1}, \mathbf{b}_{2}, \mathbf{b}_{1}+\mathbf{b}_{2}$ и им обратные (рис. 11).

Концы векторов А, C, Е и B, D, F образуют два симметрично пересекающихся треугольника, образующих первую зону Бриллюэна в виде правильного шестиугольника с особыми точками М и К. Из геометрических построений для стороны зоны Бриллюэна получаем $2 M K=4 \pi / 3 a_{0} \sqrt{3}$.

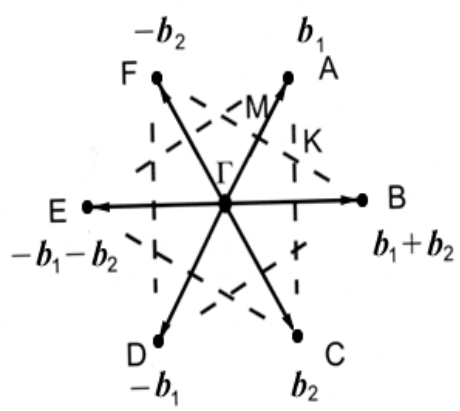

Рис. 11. Построение первой зоны Бриллюэна

Сопоставление прямой и обратной решеток показано на рис. 12.
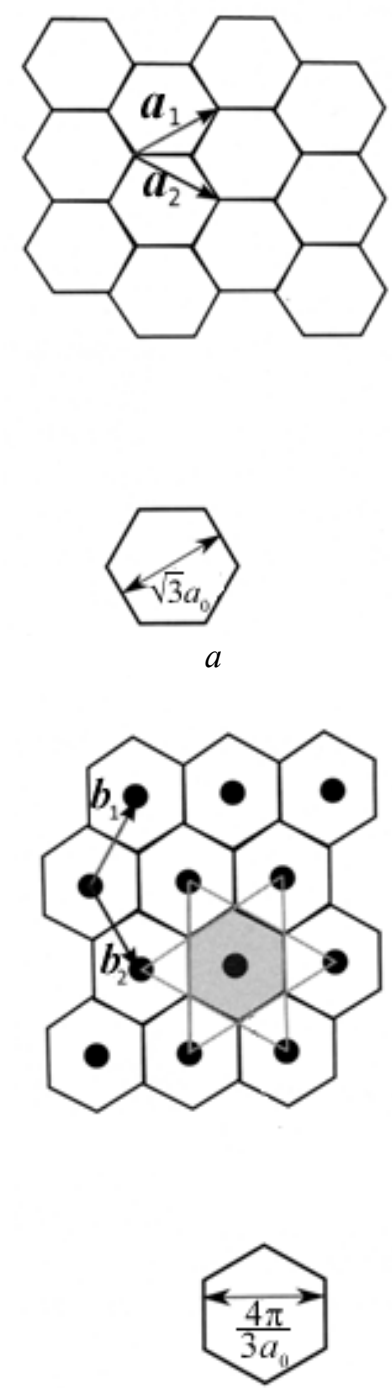

6

Рис. 12. Решетки графена: $a$ - прямая; $\sigma$ - обратная; первая зона Бриллюэна затенена

Обращает на себя внимание взаимное расположение векторов трансляции обеих решеток:

$$
\begin{aligned}
& \mathbf{b}_{1} \perp \mathbf{a}_{2} \\
& \mathbf{b}_{2} \perp \mathbf{a}_{1}
\end{aligned},
$$

что следует из (85). 


\section{Приложение II. Термоэлектрические коэффициенты для графена \\ В приведенных ниже формулах предпо- лагается линейная дисперсия (24). Число мод дается выражением (34). Рассеяние описывается степенным законом}

$$
\lambda(E)=\lambda_{0}\left(\frac{E}{k T}\right)^{r} .
$$

Положение уровня Ферми $E_{F}$ относительно дна зоны проводимости $E_{C}$ определяется параметром

$$
\eta_{F}=\frac{E_{F}-E_{C}}{k T} .
$$

Проводимость $G=\sigma W / L$ приведена в $C u$ менсах: $[\sigma]=1 C$. Аналогично для остальных удельных термоэлектрических коэффициентов (5) (8): $s_{T}=S_{T} L / W ; \kappa_{0}=K_{0} L / W ; \kappa=K L / W$.

Интегралы Ферми - Дирака порядка $j$ определяются следующим образом [31]:

$$
\mathfrak{I}_{j}\left(\eta_{F}\right)=\frac{1}{\Gamma(j+1)} \int_{0}^{\infty} \frac{\eta^{j}}{\exp \left(\eta-\eta_{F}\right)+1} d \eta .
$$

\section{1. Баллистический режим}

$$
\begin{gathered}
G^{\text {ball }}=W \frac{2 q^{2}}{h}\left(\frac{2 k T}{\pi \hbar v_{F}}\right)\left[\mathfrak{I}_{0}\left(\eta_{F}\right)+\mathfrak{I}_{0}\left(-\eta_{F}\right)\right] ; \\
S^{\text {ball }}=-\frac{k}{q}\left\{\frac{2\left[\mathfrak{I}_{1}\left(\eta_{F}\right)-\mathfrak{I}_{1}\left(-\eta_{F}\right)\right]}{\mathfrak{I}_{0}\left(\eta_{F}\right)+\mathfrak{I}_{0}\left(-\eta_{F}\right)}-\eta_{F}\right\} ; \\
S_{T}^{\text {ball }}=-W \frac{2 q^{2}}{h}\left(\frac{2 k T}{\pi \hbar v_{F}}\right)\left(\frac{k}{q}\right)\left\{2\left[\mathfrak{I}_{1}\left(\eta_{F}\right)-\mathfrak{I}_{1}\left(-\eta_{F}\right)\right]-\eta_{F}\left[\mathfrak{I}_{0}\left(\eta_{F}\right)+\mathfrak{I}_{0}\left(-\eta_{F}\right)\right]\right\} ; \\
K^{\text {ball }}=W T \frac{2 q^{2}}{h}\left(\frac{2 k T}{\pi \hbar v_{F}}\right)\left(\frac{k}{q}\right)^{2}\left\{6\left[\mathfrak{I}_{2}\left(\eta_{F}\right)+\mathfrak{I}_{2}\left(-\eta_{F}\right)\right]-\frac{4\left[\mathfrak{I}_{1}\left(\eta_{F}\right)-\mathfrak{I}_{1}\left(-\eta_{F}\right)\right]^{2}}{\mathfrak{I}_{0}\left(\eta_{F}\right)+\mathfrak{I}_{0}\left(-\eta_{F}\right)}\right\} ; \\
\times\left\{6\left[\mathfrak{I}_{2}\left(\eta_{F}\right)+\mathfrak{I}_{2}\left(-\eta_{F}\right)\right]-4 \eta_{F}\left[\mathfrak{I}_{1}\left(\eta_{F}\right)-\mathfrak{I}_{1}\left(-\eta_{F}\right)\right]+\eta_{F}^{2}\left[\mathfrak{I}_{0}\left(\eta_{F}\right)+\mathfrak{I}_{0}\left(-\eta_{F}\right)\right]\right\} .
\end{gathered}
$$

\section{2. Диффузионный режим}

$$
\begin{gathered}
G^{\text {diff }}=W \frac{2 q^{2}}{h}\left(\frac{2 k T}{\pi \hbar v_{F}}\right)\left(\frac{\lambda_{0}}{L}\right) \times \\
\times \Gamma(r+2)\left[\mathfrak{I}_{r}\left(\eta_{F}\right)+\mathfrak{I}_{r}\left(-\eta_{F}\right)\right] \\
S^{\text {diff }}=-\frac{k}{q}\left\{\frac{(r+2)\left[\mathfrak{I}_{r+1}\left(\eta_{F}\right)-\mathfrak{J}_{r+1}\left(-\eta_{F}\right)\right]}{\mathfrak{J}_{r}\left(\eta_{F}\right)+\mathfrak{I}_{r}\left(-\eta_{F}\right)}-\eta_{F}\right\} ; \\
S_{T}^{\text {diff }}=-W \frac{2 q^{2}}{h} \frac{k}{q}\left(\frac{2 k T}{\pi \hbar v_{F}}\right)\left(\frac{\lambda_{0}}{L}\right) \times \\
\times\left\{\Gamma(r+3)\left[\mathfrak{J}_{r+1}\left(\eta_{F}\right)-\mathfrak{I}_{r+1}\left(-\eta_{F}\right)\right]-\eta_{F} \Gamma(r+2)\left[\mathfrak{J}_{r}\left(\eta_{F}\right)+\mathfrak{J}_{r}\left(-\eta_{F}\right)\right]\right\} ;
\end{gathered}
$$

$K=W T \frac{2 q^{2}}{h}\left(\frac{k}{q}\right)^{2}\left(\frac{2 k T}{\pi \hbar v_{F}}\right)\left(\frac{\lambda_{0}}{L}\right) \Gamma(r+3) \times$

$\times\left\{(r+3)\left[\mathfrak{I}_{r+2}\left(\eta_{F}\right)+\mathfrak{I}_{r+2}\left(-\eta_{F}\right)\right]-\frac{(r+2)\left[\mathfrak{I}_{r+1}\left(\eta_{F}\right)-\mathfrak{I}_{r+1}\left(-\eta_{F}\right)\right]^{2}}{\mathfrak{I}_{r}\left(\eta_{F}\right)+\mathfrak{I}_{r}\left(-\eta_{F}\right)}\right\} ;$

$K_{0}=W T \frac{2 q^{2}}{h}\left(\frac{k}{q}\right)^{2}\left(\frac{2 k T}{\pi \hbar v_{F}}\right)\left(\frac{\lambda_{0}}{L}\right) \times$

$\times\left\{\begin{array}{l}\Gamma(r+4)\left[\mathfrak{J}_{r+2}\left(\eta_{F}\right)+\mathfrak{J}_{r+2}\left(-\eta_{F}\right)\right]-2 \eta_{F} \Gamma(r+3)\left[\mathfrak{J}_{r+1}\left(\eta_{F}\right)+\mathfrak{J}_{r+1}\left(-\eta_{F}\right)\right]+ \\ +\eta_{F}^{2} \Gamma(r+2)\left[\mathfrak{J}_{r}\left(\eta_{F}\right)+\mathfrak{J}_{r}\left(-\eta_{F}\right)\right]\end{array}\right\}$.

\section{2. Выводы}

На примере графена - материала, волнующего и физиков и химиков и технологов практически во всех областях творческой деятельности, показано как работает современная теория электрической проводимости и теплопроводности Ландауэра Датты - Лундстрома, подродно изложенная в предыдущих публикациях [1, 2, 31, 34]. В частности, вычислена плотность электронных состояний и носителей тока в графене, число мод и максимальная проводимость графена, плотность фононных состояний. Оценен сравнительный вклад электронов и фононов в теплопроводность графена. В справочных целях дана сводка термоэлектрических коэффициентов для графена в баллистическом и диффузионном режимах проводимости со степенным законом рассеяния, которые могут быть использованы при решении разнообразных задач графеновых технологий.

В основу настоящего обзора положены лекции Марка Лундстрома «Near-Equilibrium Transport: Fundamentals and Applications» [29], Суприе Датты «Fundamentals of Nanoelectronics, Part I: Basic Concepts» [39] и Тимоти Фишера «Thermal Energy at the Nanoscale» [36], прочитанных в 2011-2013 годах в рамках инициативы Purdue University/nanoHUB-U [www.nanohub.org/u], а также наши работы $[26,40]$.

\section{3. Благодарности}

Благодарю Н. Е.Кругляк за помощь в подготовке рукописи к печати.

\section{Литература}

1. Kruglyak, Yu. Landauer-Datta-Lundstrom Generalized Transport Model for Nanoelectronics [Text] / Yu. Kruglyak // Journal of Nanoscience. - 2014. - P. 1-15. doi: $10.1155 / 2014 / 725420$

2. Кругляк, Ю. А. Обобщенная модель электронного транспорта Ландауэра-Датты-Лундстрома [Текст] / Ю. А. Кругляк // Nanosystems, Nanomaterials, Nanotechnologies. - 2013. - Vol. 11, Issue 3. - P. 519-549. Erratum: ibid. - 2014. - Vol. 12, Issue 2. - P. 415.

3. Стріха, М. В. Фізика графену: стан і перспективи [Текст] / М. В. Стріха // Sensor Electronics Microsys. Tech. -2010 . - T. 7, № 3. - C. 5-13.

4. Geim, A. K. Graphene: Status and Prospects [Text] / A. K. Geim // Science. - 2009. - Vol. 324, Issue 5934. - P. 1530-1534. doi: 10.1126/science.1158877 
5. Novoselov, K. S. Beyond the wonder material [Text] / K. S. Novoselov // Physics World. - 2009. - Vol. 22, Issue 8. - P. 27-30.

6. Лозовик, Ю. Е. Коллективные электронные явления в графене [Текст] / Ю. Е. Лозовик, С. П. Меркулова, А. А. Соколик // Успехи физических наук. - 2008. - Т. 178, № 7. - С. 757-776. doi: 10.3367/ufnr.0178.200807h.0757

7. Морозов, С. В. Электронный транспорт в графене [Текст] / С. В. Морозов, К. С. Новоселов, А. К. Гейм // Успехи физических наук. - 2008. - Т. 178, № 7. C. 776-780. doi: 10.3367/ufnr.0178.200807i.0776

8. Tsuneya, A. Physics of Graphene. Zero-Mode Anomalies and Roles of Symmetry [Text] / A. Tsuneya // Progress of Theoretical Physics Supplement. - 2008. Vol. 176. - P. 203-226. doi: 10.1143/ptps.176.203

9. Geim, A. K. The Rise of Graphene [Text] / A. K. Geim, K. S. Novoselov // Nature Materials. - 2007. Vol. 6. - P. 183-191. doi: 10.1038/nmat1849

10. McClure, J. W. Diamagnetism of Graphite [Text] / J. W. McClure // Physical Review. - 1956. - Vol. 104, Issue 3. - P. 666-671. doi: 10.1103/physrev.104.666

11. Slonczewski, J. C. Band Structure of Graphite [Text] / J. C. Slonczewski, P. R. Weiss // Physical Review. 1958. - Vol. 109, Issue 2. - P. 272-279. doi: 10.1103/ physrev.109.272

12. Ando, T. Theory of electronic states and transport in carbon nanotubes [Text] / T. Ando // Journal of the Physical Society of Japan. - 2005. - Vol. 74, Issue 3. - P. 777-817. doi: 10.1143/jpsj.74.777

13. Shon, N. H. Quantum transport in two-dimensional graphite system [Text] / N. H. Shon, T. Ando // Journal of the Physical Society of Japan. - 1998. - Vol. 67, Issue 7. P. 2421-2429. doi: 10.1143/jpsj.67.2421

14. Peres, N. M. R. Phenomenological study of the electronic transport coefficients of graphene [Text] / N. M. R. Peres, J. M. B. Lopes dos Santos, T. Stauber // Physical Review B. - 2007. - Vol. 76, Issue 7. doi: 10.1103/physrevb.76.073412

15. Zhu, W. Carrier scattering, mobilities, and electrostatic potential in monolayer, bilayer, and trilayer graphene [Text] / W. Zhu, V. Perebeinos, M. Freitag, P. Avouris // Physical Review B. - 2009. - Vol. 80, Issue 23. P. 235-402. doi: /10.1103/physrevb.80.235402

16. Perebeinos, V. Inelastic scattering and current saturation graphene [Text] / V. Perebeinos, P. Avouris // Physical Review B. - 2010. - Vol. 81, Issue 19. doi: 10.1103/physrevb.81.195442

17. Das Sarma, S. Electronic transport in twodimensional graphene [Text] / S. Das Sarma, S. Adam, E. H. Hwang, E. Rossi // Reviews of Modern Physics. - 2011.Vol. 83, Issue 2. - P. 407-470. doi: 10.1103/revmodphys. 83.407

18. Novoselov, R. S. Electric Field Effect in Atomically Thin Carbon Films [Text] / R. S. Novoselov, A. K. Geim, S. V. Morozov, D. Jiang, Y. Zhang, S. V. Dubonos, I. V. Grigorieva, A. A. Firsov // Science. - 2004. - Vol. 306, Issue 5659. - P. 666-669. doi: 10.1126/science.1102896

19. Morozov, S. V. Two-dimensional electron and hole gases at the surface of graphite [Text] / S. V. Morozov, K. S. Novoselov, F. Schedin, D. Jiang, A. A. Firsov, A. K. Geim // Physical Review B. - 2005. - Vol. 72, Issue 20. doi: 10.1103/physrevb.72.201401

20. Novoselov, R. S. Two-Dimensional Gas of Massless Dirac Fermions in Graphene [Text] / R. S. Novoselov, A. K. Geim, S. V. Morozov, D. Jiang, M. I. Katsnelson, I. V. Grigorieva, S. V. Dubonos, A. A. Firsov // Nature. 2005. - Vol. 438, Issue 7065. - P. 197-200.

Doi: $10.1038 /$ nature04233
21. Zhang, Y. Experimental observation of the quantum Hall effect and Berry's phase in grapheme [Text] / Y. Zhang, Y.-W. Tan, H. L. Stormer, P. Kim // Nature. - 2005. Vol. 438, Issue 7065. - P. 201-204. doi: 10.1038/nature04235

22. Laughlin, R. B.Condensed Matter Theory (II): Graphene Band Structure [Electronic resource] / R. B. Laughlin. - Graphene Density of States. - Available at: http://large.stanford.edu/courses/

23. Supriyo Datta. Graphene Bandstructures [Electronic resource] / Purdue University, 2008. - Available at: www.nanohub.org/resources/5710

24. Supriyo Datta. Graphene Density of States I [Electronic resource] / Purdue University, 2008. - Available at: www.nanohub.org/resources/5721

25. Supriyo Datta. Graphene Density of States II [Electronic resource] / Purdue University, 2008. - Available at: www.nanohub.org/resources/5722

26. Кругляк, Ю. А. Методические аспекты расчета зонной структуры графена с учетом $\sigma$-остова. Теоретические основы [Текст] / Ю. А. Кругляк, Н. Е. Кругляк // Вісник Одеського державного екологічного університету. - 2012. - № 13. - С. 207-218.

27. Mark Lundstrom. Sums in k-space/Integrals in Energy Space [Electronic resource] / Purdue University, 2009. - Available at: www.nanohub.org/resources/7296

28. Berdebes, D. Lecture Notes on Low Bias Transport in Graphene: An Introduction [Electronic resource] / D. Berdebes, T. Low, M. Lundstrom. - Purdue University, 2009. - Available at: www.nanohub.org/resources/7435

29. Lundstrom, M. Near-Equilibrium Transport: Fundamentals and Applications [Electronic resource] / M. Lundstrom, C. Jeong. - Hackensack, New Jersey: World Scientific Publishing Company, 2013. - Available at: www.nanohub.org/resources/11763

30. Кругляк, Ю. О. Уроки наноелектроніки: ефект Холла i вимірювання електрохімічних потенціалів у концепції «знизу - вгору» [Текст] / Ю. О. Кругляк, М. В. Стріха // Сенсорна електроніка і мікросистемні технології. - 2014. - Т. 11, № 1. - С. 5-27.

31. Кругляк, Ю. А. От баллистической проводимости к диффузионной в транспортной модели Ландауэра-Датты-Лундстрома [Текст] / Ю. А. Кругляк // Nanosystems, Nanomaterials, Nanotechnologies. - 2013. T. 11, № 4. - С. 655-677.

32. Кругляк, Ю. А. Уроки наноэлектроники. 3. Электронная проводимость и моды проводимости в концепции «снизу - вверх» [Текст] / Ю. А. Кругляк, Н. Е. Кругляк // Физическое образование в вузах. - 2013. T. 19, № 3. - С. 99-110.

33. Кругляк, Ю. А. Уроки наноэлектроники. 2. Модель упругого резистора и новая формулировка закона Ома в концепции «снизу - вверх» [Текст] / Ю. А. Кругляк, Н. Е. Кругляк // Физическое образование в вузах. - 2013. T. 19, № 2. - C. 161-173.

34. Kruglyak, Yu. A. Heat transfer by phonons in Landauer-Datta-Lundstrom approach, Proceedings of the International Conference [Text] / Yu. A. Kruglyak // Nanomaterials: Applications and Properties. - 2014. - Vol. 3, Issue 2. - P. 5.

35. Singh, D. Spectral phonon conduction and dominant scattering pathways in graphene [Text] / D. Singh, J. Y. Murthy, T. S. Fisher // Journal of Applied Physics. 2011. - Vol. 110, Issue 9. doi: 10.1063/1.3656451

36. Fisher, T. S. Thermal Energy at the Nanoscale [Electronic resource] / T. S. Fisher // Hackensack, New Jersey: World Scientific Publishing Company, 2013. - Available at: www.nanohub.org/courses/2

37. Das Sarma, S. Electronic transport in two-dimensional graphene [Text] / S. Das Sarma, S. Adam, E. H. Hwang, 
E. Rossi // Reviews of Modern Physics. - 2011. - Vol. 83, Issue 2. - P. 407-470. doi: 10.1103/revmodphys.83.407

38. Kim, R. S. Physics and Simulation of Nanoscale Electronic and Thermoelectric Devices [Text] / R. S. Kim. West Lafayette: Purdue University, 2011. - 220 p.

39. Supriyo Datta, Lessons from Nanoelectronics: A New Perspective on Transport [Electronic resource] / Hackensack, New Jersey: World Scientific Publishing Company, 2012. - Available at: www.nanohub.org/ courses/FoN1

40. Кругляк, Ю. О. Уроки наноелектроніки: Метод нерівноважних функцій Гріна у матричному зображенні. II. Модельні транспортні задачі [Текст] / Ю. О. Кругляк, М. В. Стріха // Сенсорна електроніка і мікросистемні технології. - 2013. - Т. 10, № 4. - С. 5-22.

\section{References}

1. Kruglyak, Yu. (2014). Landauer-Datta-Lundstrom Generalized Transport Model for Nanoelectronics, Journal of Nanoscience, 15. doi: 10.1155/2014/725420

2. Kruglyak, Yu. A. (2013). Landauer-Datta-Lundstrom Generalized Electron Transport Model for Nanoelectronics. Nanosystems, Nanomaterials, Nanotechnologies, 11 (3), 519549. Erratum: ibid, (2014). 12 (2), 415.

3. Strikha, M. V. (2010). Physics of Graphene: Status and Perspectives. Sensor Electronics Microsys. Tech., 7 (3), 5-13.

4. Geim, A. K. (2009). Graphene: Status and Prospects. Science, 324 (5934), 1530-1534.

doi: $10.1126 /$ science. 1158877

5. Novoselov, K. S. (2009). Beyond the wonder material. Physics World, 22 (8), 27-30.

6. Lozovik, Yu. E., Merkulova, S. P., Sokolik, A. A. (2008). Collective electron phenomena in graphene. Physics Uspekhi, 51, 727-744. doi: 10.3367/ufnr.0178.200807h.0757

7. Morozov, S. V., Novoselov, K. S., Geim, A. K. (2008). Electronic transport in graphene. Physics Uspekhi, 51, 744-748 doi: 10.3367/ufnr.0178.200807i.0776

8. Tsuneya, A. (2008). Physics of Graphene. ZeroMode Anomalies and Roles of Symmetry. Progress of Theoretical Physics Supplement, 176, 203-226.

doi: $10.1143 /$ ptps. 176.203

9. Geim, A. K., Novoselov, K. S. (2007). The Rise of Graphene. Nature Materials, 6, 183-191.

doi: 10.1038/nmat1849

10. McClure, J. W. (1956). Diamagnetism of Graphite. Physical Review, 104 (3), 666-671.

doi: 10.1103/physrev.104.666

11. Slonczewski, J. C., Weiss, P. R. (1958). Band Structure of Graphite. Physical Review, 109 (2), 272-279. doi: 10.1103/physrev.109.272

12. Ando, T. (2005). Theory of electronic states and transport in carbon nanotubes. Journal of the Physical Society of Japan, 74 (13), 777-817. doi: 10.1143/jpsj.74.777

13. Shon, N. H., Ando, T. (1998). Quantum transport in two-dimensional graphite system. Journal of the Physical Society of Japan, 67 (7), 2421-2429. doi: 10.1143/jpsj.67.2421

14. Peres, N. M. R., Lopes dos Santos, J. M. B., Stauber, T. (2007). Phenomenological study of the electronic transport coefficients of graphene. Physical Review B, 76 (7). doi: 10.1103/physrevb.76.073412

15. Zhu, W., Perebeinos, V., Freitag, M., Avouris, P. (2009). Carrier scattering, mobilities, and electrostatic potential in monolayer, bilayer, and trilayer graphene, Physical Review B, 80 (23), 235-402. doi: /10.1103/physrevb.80.235402

16. Perebeinos, V., Avouris, P. (2010). Inelastic scattering and current saturation graphene. Physical Review B, 81 (19). doi: 10.1103/physrevb.81.195442

17. Das Sarma, S., Adam, S., Hwang, E. H., Rossi, E. (2011). Electronic transport in two-dimensional graphene.
Reviews of Modern Physics, 83 (2), 407-470. doi: 10.1103/revmodphys.83.407

18. Novoselov, R. S., Geim, A. K., Morozov S. V., Jiang, D., Zhang, Y., Dubonos, S. V., Grigorieva, I. V., Firsov, A. A. (2004). Electric Field Effect in Atomically Thin Carbon Films. Science, 306 (5659), 666-669.

doi: 10.1126/science.1102896

19. Morozov, S. V., Novoselov, K. S., Schedin, F., Jiang, D., Firsov, A. A., Geim, A. K. (2005). Two-dimensional electron and hole gases at the surface of graphite. Physical Review B, 72 (20). doi: 10.1103/physrevb.72.201401

20. Novoselov, R. S., Geim, A. K., Morozov, S. V., Jiang, D., Katsnelson, M. I., Grigorieva, I. V., Dubonos, S. V., Firsov, A. A. (2005). Two-Dimensional Gas of Massless Dirac Fermions in Graphene. Nature, 438 (7065), 197-200. doi: 10.1038/nature04233

21. Zhang, Y., Tan, Y.-W., Stormer, H. L., Kim, P. (2005). Experimental observation of the quantum Hall effect and Berry's phase in grapheme. Nature, 438 (7065), 201-204. doi: 10.1038/nature04235

22. Laughlin, R. B. Condensed Matter Theory (II): Graphene Band Structure. Graphene Density of States. Available at: http://large.stanford.edu/courses/

23. Supriyo Datta. Graphene Bandstructures (2008). Purdue University. Available at: www.nanohub.org/ resources/5710

24. Supriyo Datta. Graphene Density of States I (2008). Purdue University. Available at: www.nanohub.org/ resources $/ 5721$

25. Supriyo Datta. Graphene Density of States II (2008). Purdue University. Available at: www.nanohub.org/ resources/5722

26. Kruglyak, Yu. A., Kruglyak, N. E. (2012). Calculation of graphene band structure. Methodological and theoretical basis. Visnyk Odessa State Ecolog. Univ., 13, 207-218.

27. Lundstrom, M. (2009). Sums in k-space/Integrals in Energy Space. Purdue University. Available at: www.nanohub.org/resources/7296

28. Berdebes, D., Low, T., Lundstrom, M. (2009). Lecture Notes on Low Bias Transport in Graphene: An Introduction. Purdue University. Available at: www.nanohub.org/resources/7435

29. Lundstrom, M., Jeong, C. (2013). Near-Equilibrium Transport: Fundamentals and Applications. Hackensack, New Jersey: World Scientific Publishing Company. Available at: www.nanohub.org/resources/11763

30. Kruglyak, Yu. A., Strikha, M. V. (2014). Lessons of nanoelectronics: Hall effect and measurement of electrochemical potentials within «bottom-up» approach, Sensor Electronics Microsys. Tech., 11 (1), 5-27.

31. Kruglyak, Yu. A. (2013). From Ballistic Conductivity to Diffusional in the Landauer-Datta-Lunstrom Transport Model. Nanosystems, Nanomaterials, Nanotechnologies, 11 (4), 655-677.

32. Kruglyak, Yu. A., Kruglyak, N. E. (2013). Lessons of nanoelectronics. 3. Electronic conductivity and conductivity modes by «bottom - up» approach, Physics in Higher Education, 19 (3), 99-110.

33. Kruglyak, Yu. A., Kruglyak, N. E. (2013). Lessons of nanoelectronics. 2. Elastic resistor model and new Ohm's law by «bottom - up» approach, Physics in Higher Education, 19 (2), 161-173.

34. Kruglyak, Yu. A. (2014).Heat transfer by phonons in Landauer-Datta-Lundstrom approach, Proceedings of the International Conference. Nanomaterials: Applications and Properties", 3 (2), 5.

35. Singh, D., Murthy, J. Y., Fisher, T. S. (2011). Spectral phonon conduction and dominant scattering pathways in graphene. Journal of Applied Physics, 110 (9).

doi: 10.1063/1.3656451 
36. Fisher, T. S. (2013). Thermal Energy at the Nanoscale. Hackensack, New Jersey: World Scientific Publishing Company. Available at: www.nanohub.org/courses/2

37. Das Sarma, S., Adam, S., Hwang, E. H., Rossi, E. (2011). Electronic transport in two-dimensional graphene. Reviews of Modern Physics, 83 (2), 407-470.

doi: $10.1103 /$ revmodphys. 83.407

38. Kim, R. S. (2011). Physics and Simulation of Nanoscale Electronic and Thermoelectric Devices. West
Lafayette: Purdue University, 220.

39. Supriyo Datta, Lessons from Nanoelectronics: A New Perspective on Transport (2012). Hackensack, New Jersey: World Scientific Publishing Company. Avaialble at: www.nanohub.org/courses/FoN1

40. Kruglyak, Yu. A., Strikha, M. V. (2013). Lessons of nanoelectronics: Non-equilibrium Green's functions method in matrix representation. II. Model transport problems, Sensor Electronics Microsys. Tech., 10 (4), 5-22.

Рекомендовано до публікації д-р фіз.-мат. наук Глушков О. В. Дата надходження рукопису 15.01.2015

Кругляк Юрий Алексеевич, доктор химических наук, профессор, кафедра информационных технологий, Одесский государственный экологический университет, ул. Львовская, 15, Одесса, Украина, 65016

E-mail: quantumnet@yandex.ua

УДК 53.07+57.043

DOI: 10.15587/2313-8416.2015.37505

\section{МИКРООБЪЁМНЫЙ СКАНИРУЮЩИЙ ТЕНЗОДИЛАТОМЕТР ДЛЯ БИОФИЗИЧЕСКИХ ИССЛЕДОВАНИЙ}

\section{(c) А. И. Осецкий, С. С. Севастьянов}

В настоящей статье рассматривается принципиально новый способ построения высокоэффективных объёмных дилатометров, предназначенных для исследования кинетики процессов, протекающих в жидкостях в широком диапазоне температур и давлений. Конструкиия ячеек таких дилатометров минимизирует температурные градиенты в исследуемых растворах при охлаждении или нагреве и позволяет существенно повысить их разрешаюшую способность

Ключевые слова: кинетика, объёмный эффект, дилатометр, сильфон, ячейка, криопротектор, разрешающая способность

In this paper we consider a fundamentally new way of building the high-performance volumetric dilatometers designed to study the kinetics of the processes occurring in liquids in a wide range of temperatures and pressures. The construction of such cell dilatometers minimizes thermal gradients in the solutions or in cooling and heating and allows significantly improve their minimum grade value

Keywords: kinetics, volume effect, dilatometer, bellows element, cell, cryoprotectant, minimum grade value

\section{1. Введение}

Анализ физических методов исследования активируемых процессов в биологических системах показывает, что разработка приборов для прецизионных измерений их объёма в широком диапазоне температур и давлений является актуальной проблемой современного биофизического приборостроения. Во-первых, это связано с тем, что объём V исследуемой макроскопической системы входит в уравнение состояния этой системы как один из основных термодинамических параметров [1]. Вследствие этого по изменению объёма можно судить не только о кинетике таких кооперативных явлений, как фазовые и конформационные превращения, химические и биохимические реакции, но и о влиянии на эти явления различных внешних воздействий и физико-химических свойств среды. Во-вторых, методы микрообъёмной дилатометрии при исследовании биологических систем и растворов эффективны в очень широких диапазонах. Это особенно наглядно проявляется при низкотемпературных биофизи-ческих исследованиях. Так, после начала кристал-лизации в охлаждаемых биологи- ческих системах становится практически неприменимой для их исследования спектроскопия как в инфракрасном, так и в ультрафиолетовом диапазонах [2]. С понижением температуры уменьшаются по абсолютной величине тепловые эффекты, сопровождающие различные биохимические процессы в консервируемых био-объектах [3], что затрудняет применение традиционных калориметрических методик. Охлаждение биологических систем сопровождается значительным падением подвижности введённых в них спиновых и ядерных меток, что приводит к снижению эффективности методов ЭПР и ЯМР [4]. В то же время большинство процессов, протекающих в охлаждаемых биологических системах, сопровождается значительными изменениями их объёма, что обуславливает целесообразность разработки и усовершенствования дилатометрических методов исследования указанных процессов.

\section{2. Литературный обзор}

В основе существующих способов измерения объёмных эффектов лежит принцип их трансформации в линейные перемещения, фиксируемые 